\title{
Neutrophil extracellular traps in vasculitis, friend or foe?
}

Daniel Söderberg and Mårten Segelmark

The self-archived postprint version of this journal article is available at Linköping University Institutional Repository (DiVA):

http:// urn.kb.se/ resolve?urn=urn:nbn:se:liu:diva-147444

N.B.: When citing this work, cite the original publication.

Söderberg, D., Segelmark, M., (2018), Neutrophil extracellular traps in vasculitis, friend or foe?,

Current Opinion in Rheumatology, 30(1), 16-23. https:// doi.org/ 10.1097/ BOR.0000000000000450

Original publication available at:

https:// doi.org/ 10.1097/BOR.0000000000000450

Copyright: Lippincott, Williams \& Wilkins

http:// www.lww.com/ 


\section{$1 \quad$ Neutrophil extracellular traps in vasculitis, friend or foe?}

2 Daniel Söderberg ${ }^{1}$ and Mårten Segelmark ${ }^{1,2}$

$3 \quad{ }^{1}$ Department of Medical and Health Sciences and ${ }^{2}$ Department of Nephrology, Linköping

$4 \quad$ University, Linköping, Sweden

5 Corresponding author: Daniel Söderberg

6 Email: daniel.soderberg@liu.se

7 Address: Department of Medical and Health Sciences, Linköping University, 58185

8 Linköping, Sweden

9 Telephone: $+46-101031515$ 
Abstract

Purpose of review: Neutrophil extracellular traps (NETs) can be found at the sites of vascular lesions and in the circulation of patients with active small vessel vasculitis. Neutrophils from vasculitis patients release more NETs in vitro, and NETs have properties that can harm the vasculature both directly and indirectly. There are several ways to interfere with NET formation, which open for new therapeutic options. However, there are several types of NETs and different mechanisms of NET formation, and these might have different effects on inflammation. Here we review recent findings regarding the pathogenesis and therapeutic potentials of NETs in vasculitis.

Recent findings: Experimental mouse models support a role for NETs in promoting vascular damage, where histones and mitochondrial DNA appear to be driving forces. However, impaired formation of NETs in an SLE-like mouse model leads to more severe disease, suggesting that NETs can be important in limiting inflammation. Studies on drug-induced vasculitis reveal that levamisole can induce NETosis via muscarinic receptors, predisposing for the generation of autoantibodies, including anti-neutrophil cytoplasmic autoantibodies (ANCA). This supports the notion that NETs can bridge the innate and adaptive immune systems.

Summary: NETs can participate in the pathogenesis of vasculitis, but in some models there also seem to be protective effects of NETs. This complexity needs further evaluation with experimental models that are as specific as possible for human primary vasculitis. 
Keywords

Vasculitis, Neutrophil extracellular traps (NETs), ANCA, Autoantigens, Inflammation

\section{Introduction}

The formation of neutrophil extracellular traps (NETs) was initially described as a mechanism to ensnare and kill invading microorganisms (1), but in recent years NETs have attracted increased attention in a wide variety of medical conditions such as cancer, thromboembolism, arteriosclerosis, and autoimmune diseases (2). Kessenbrock and coworkers suggested a role for NETs in the pathogenesis of vasculitis in 2009 (3), and since then there has been a growing body of literature on the connections between NETs and vasculitis. Here we give an overview of recent advances pertaining to altered NET formation in vasculitis, the relation between NETs and vascular damage, NETs as a source of autoantigens, the utility of biomarkers associated with NETs, and finally some possible therapeutic implications of NET formation in vasculitis.

\section{Definitions and nomenclature}

The formation of NETs, called NETosis, was originally proposed to be a form of programmed cell death initiated by nicotinamide adenine dinucleotide phosphate (NADPH) oxidase activation followed by chromatin decondensation, breakdown of the nuclear membrane, and mixing of the chromatin with granule constituents (4). The process is dependent on myeloperoxidase (MPO), neutrophil elastase, and peptidyl arginine deiminase (PAD) $4(5,6)$ and results in the extrusion of a tangle of DNA decorated with citrullinated histones and other proinflammatory molecules (7). However, subsequent research has questioned whether each step in this pathway is necessary for NETosis. It was, for example, recently shown that saliva can induce NETosis independently of NADPH oxidase and 
neutrophil elastase (8). Neutrophils are not the only cells that can extrude extracellular traps (ETs), and eosinophils, basophils, mast cells, and monocytes also have such capacity $(9,10)$, and the term ETosis has been coined as a general term for cells releasing ETs. It has been shown that extrusion of NETs is not necessarily associated with cell death, and today many authors distinguish between NETosis involving cell death (suicidal NETosis) and NETosis where the neutrophils remain viable (vital NETosis). NETs released during vital NETosis can consist of nuclear or mitochondrial (mt) DNA and can be released in an NADPH-oxidase and/or reactive oxygen species (ROS)-independent manner (10-13).

Primary systemic vasculitis encompasses a wide variety of diseases with idiopathic vascular inflammation as their common defining feature. According to the current nomenclature, they are divided into groups based on the size of the vessels that are predominantly affected in the individual diseases (14). The small-vessel vasculitides are further grouped according to immunofluorescence findings of biopsies into immune-complex vasculitides and pauciimmune vasculitides. The latter are also referred to as anti-neutrophil cytoplasmic autoantibody (ANCA)-associated vasculitis (AAV) because of their relationship to ANCA, and this group contains the diseases granulomatosis with polyangiitis (GPA, formerly Wegener's granulomatosis), microscopic polyangiitis (MPA), and eosinophilic granulomatosis with polyangiitis (EGPA, formerly Churg-Strauss syndrome) (14). More common than primary vasculitis is vasculitis as a complicating feature of other autoimmune diseases, infections, malignancies, or adverse drug reactions (15).

\section{Vasculitis is associated with increased NETosis}

The pathogenesis varies between different forms of vasculitis, but at least in small-vessel vasculitis neutrophils have a prominent role. Neutrophils produce ROS and release 
destructive enzymes, and they attract other players to the scene through the production of cytokines and chemokines. It is often difficult to distinguish the contribution of NETs relative to activation, degranulation, and other forms of neutrophil cell death than NETosis. There are several investigations showing increased NETosis in active vasculitis, and NETs and/or remnants of NETs can be found both in the affected tissues and in the blood circulation of AAV patients (16). Co-expression of granule proteins (such as MPO and neutrophil elastase) and chromatin (primarily citrullinated histone 3) is often considered as evidence of NETosis. However, one needs to be aware when screening for NETs that they can also be mitochondrial-derived and thus would not contain histones.

NETs in AAV were first reported on in kidney biopsies (3), which was later confirmed by others (17-20), and then also in skin specimens $(21-23)$ and in thrombi $(20,24)$ of these patients. Neuropathy is another common feature of vasculitis, and NETs were recently shown to also be common in nerve biopsies from AAV patients, but not seen in patients with nonvasculitic demyelinating neuropathy (25). More NETs were seen in ANCA-positive MPA patients compared to ANCA-negative MPA patients and in patients with vasculitis secondary to rheumatoid arthritis (25). Increased levels of breakdown fragments of NETs (NET remnants) in the circulation have also been reported in vasculitis $(3,26,27)$.

In vitro studies on neutrophils from AAV patients show that they are more prone to undergo spontaneous NETosis $(18,26,28)$ and are more responsive to NET-inducing stimuli (29). Similar findings have been reported in other autoimmune diseases were NETosis is implicated in the pathogenesis (30-32). It was recently also revealed that NETosis is negatively regulated by interaction between plexin B2 on endothelial cells and semaphorin 
4D on neutrophils, and NETosis in humans cells can be inhibited in vitro by recombinant plexin B2 (33). Interestingly, neutrophils from AAV patients exhibit reduced expression of semaphorin 4D compared with healthy controls (33), which could be an explanation for the increased amount of NETs in these patients.

\section{Serum, immune complexes, and autoantibodies from vasculitis patients induce NETosis}

\section{in vitro}

In vitro studies have shown that IgG and serum from AAV patients can stimulate neutrophils from healthy controls to undergo NETosis to a greater extent than IgG and serum from healthy controls (Table 1) (3, 33-39). For ANCA IgG, this generally requires the neutrophils to be primed in order to increase the membrane expression of proteinase 3 (PR3) and MPO before they will respond to ANCA exposure by undergoing NETosis. This can, for example, be seen in recent reports that have shown an effect of PR3-ANCA IgG and MPO-ANCA IgG on NETosis after priming with high mobility group box 1 (HMGB1) (35) or tumour necrosis factor (36), respectively. The ability of MPO-ANCA IgG to induce NETosis appears to be related to antibody affinity rather than antibody levels (34), and it has been shown that patients with high-affinity MPO-ANCA IgG exhibit higher occurrence of NETs in renal biopsies than patients with low-affinity MPO-ANCA IgG (40). However, IgG-depleted serum (38) and serum from ANCA-negative patients (39) have a similar ability as whole serum to induce NETosis, and this questions the role of ANCA IgG in these experimental settings, which did not include priming of the neutrophils before stimulation.

IgG-containing immune complexes can also induce NETosis, and the most recent study showed that these complexes activate neutrophils via cross-linking of Fc gamma receptor IIIb (41). Another recent study found that heat-aggregated immune complexes from patients with 
systemic lupus erythematosus (SLE) and rheumatoid arthritis (where secondary vasculitis is common) induce NETosis, but that study did not look at receptor specificity (42). IgA immune complexes in plasma and synovial fluid from rheumatoid arthritis patients were shown to induce NETosis via Fc alpha receptor I (43). This most probably has a bearing on IgA vasculitis, a disease characterised by IgA immune complex deposition and small-vessel leucocytoclastic vasculitis (14). A recent study on patients with PR3-ANCA-associated vasculitis showed that serum PR3-ANCA IgA levels were more closely related to disease activity than PR3-ANCA IgG levels (44).

\section{NETs and vascular damage}

There are several ways in which NETosis can harm the vasculature, both directly and indirectly. The release of noxious substances such as degrading enzymes can directly induce apoptosis in endothelial cells and degrade the basement membrane (45), and histones can be toxic to endothelial cells (46). A recent study showed that endothelial cells can phagocytise NETs, but that excessive amount of NETs promotes vascular leakage by interfering with endothelial cell-cell interactions (47). The same study also showed that NETs can induce endothelial to mesenchymal transformation (EndMT) and that such cells are increased in the glomeruli both in MLR/lpr mice (a mouse model of SLE) as well as in patients with lupus nephritis (47). EndMT is important during vascular repair, but it is also connected to several disease conditions because it contributes to tissue fibrosis (48), which is a common feature in vasculitis. Indirectly, NETs promote vascular damage by activating the alternative complement pathway (49).

Renal injury is common in small vessel vasculitis, including both glomerulonephritis with crescent formation and tubulointerstitial nephritis. Recent studies have shown that NETs are 
present in glomeruli and that they contribute to glomerular injury in mouse models of glomerular vasculitis induced by anti-glomerular basement membrane (GBM) antibodies (50) or GBM antiserum (46), as well as in MLR/lpr mice that spontaneously develop SLE-like disease $(47,51)$. The role of NETs in tubulointerstitial injury was shown in a study of ischemic acute kidney injury (AKI) in mice, where epithelial tubular cells during hypoxia released histones that activated neutrophils to release NETs (52). These NETs in turn induced epithelial cell necrosis with the release of histones from these cells, thus creating a necroinflammation loop leading to enhanced tubular necrosis. This study mimics a possible scenario during excessive inflammation, with hypoxia and kidney injury, as is seen in vasculitis. Tubulointerstitial injury could also retard glomerular blood flow, thus reducing the shear stress, which has been shown to rapidly clear the glomeruli of NETotic neutrophils (50). Regarding immune complex vasculitis, NETs were shown to contribute to vessel destruction and haemorrhage in mouse skin specimens after injection of bovine serum albumin (BSA) and anti-BSA antibodies (53).

\section{NETs can be protective}

A recent study showed that saliva can induce NETs, and that this capacity is diminished in Bechet's disease, a form of primary vasculitis characterised by mouth and genital ulcers (8). The authors argued that the absence of NETs leads to diminished protection against bacteria on the mucus membranes and that this promotes ulcer formation. Other examples where reduced NETosis leads to more severe disease are mouse models of SLE (54) and gout (55). These studies suggest that NETs can act as platforms to degrade proinflammatory mediators that would otherwise drive inflammation. Additionally, NETs can impair GM-CSF/IL-4induced dendritic cell differentiation from monocytes in vitro, and can instead promote an alternatively activated macrophage phenotype (56). This subgroup of macrophages is 
important for the resolution of inflammation, which is crucial for preventing chronic inflammation.

\section{Antigen exposure in NETs promotes the production of autoantibodies}

NETs contain an array of molecular motifs that serve as targets for autoantibodies in autoimmune diseases, including double-stranded DNA in SLE (32), citrullinated peptides in rheumatoid arthritis (31), and MPO and PR3 in AAV (3). NETs can also contain alarmins, such as LL39 and HMGB1 $(57,58)$, that provide danger signals and thus reduce immunological tolerance. The strongest evidence that NETs actually serve as a source of autoantigens driving autoantibody production in vasculitis comes from studies on druginduced vasculitis. MPO-ANCA positivity is relatively common in patients treated with the anti-thyroid drug propylthiouracil (PTU), and some of these patients develop a vasculitis-like syndrome (59). Phorbol 12-myrsetate 13-acetate (PMA) in combination with the anti-thyroid drug PTU induces NETs that resist DNase I degradation, and such NETs cause the production of ANCAs and AAV-like disease in rats (60). Using a similar approach as above but in a mouse model, PMA and PTU again resulted in the production of MPO-ANCA, but did not induce disease (61), indicating that antibody formation is not sufficient to induce fullblown disease. Levamisole, a veterinary compound often found in adulterated cocaine, is also associated with ANCA formation and vasculitis-like syndromes. Contrary to PTU, levamisole directly induces NETosis in neutrophils in vitro via the stimulation of muscarinic receptors (23, 62). Also, cocaine itself is able to induce NETosis (62). Patients with cocaine/levamisole-associated autoimmunity possess IgG class autoantibodies against NET components such as neutrophil elastase (62), PR3, MPO, LL-37, and anti-nuclear antibodies (23). Further, IgG from patients with levamisole-associated autoimmunity enhance NETosis induced by cocaine or levamisole, which could possibly create a vicious circle in these patients (62). 
Monitoring of disease activity is an unmet need in vasculitis, and better monitoring will enable more efficient use of the drugs available today and will reduce the side effects of maintenance therapy. As reviewed elsewhere (16), several studies in recent years have reported on increased levels of NETs and NET-associated proteins in the circulation of AAV patients that often correlate with disease activity. However, there is no assay available today that has proven to be clinically useful. Measurements of NETs suffer from a lack of standardisation, as well as from problems with sensitivity and specificity. Because of this there are currently no general values for these parameters regarding the presence of NETs in various diseases. The fact that ANCAs of different affinities appear to vary in their NETinducing capacity encourages further studies with this approach to evaluate its usefulness to monitor disease activity (34). The capacity for serum to degrade NETs is another tempting approach, and this capacity is reduced in serum from AAV patients (34). DNase I activity did not vary with disease activity in that study, but NET degradation per se with serum from patients with various disease activity was not evaluated. Regardless of the methodological approach, further evaluation of NETs as a biomarker to monitor disease activity in AAV (alone or in combination with other parameters) requires carefully undertaken longitudinal studies.

\section{Implications for treatment}

As summarised in Table 2, several approaches have been used in recent studies to block the effect of NETs on vascular damage in different in vivo experimental models. Anti-histone treatment through anti-histone antibodies, heparin, or activated protein $\mathrm{C}$ all proved to be efficient in treating anti-GBM-induced vasculitis (46). This was also the case for the PAD inhibitor Cl-amidine (46), which inhibits the citrullination of histones that is an important 
step during suicidal NETosis. Inhibiting PAD signalling was also shown to be efficient in a mouse model of post-ischemic AKI (52). Injection of adipose tissue-derived mesenchymal stem cells (MSCs) prior to injection of BSA/anti-BSA antibodies in a model of immune complex vasculitis significantly reduced the vessel damage and the amount of NETs that were formed (53). The inhibitory mechanisms of the MSCs included phagocytosis of neutrophils and upregulation of superoxide dismutase 3, an antioxidant, both of which prevented NET formation (53). There is a plethora of other molecules that are being tested for their ability to block NETosis, and these are primarily being evaluated in vitro. A recent example is the blockade of neutrophil elastase, which has been shown to inhibit NET-induced disruption of endothelial cell-cell integrity and EndMT (48). Although inhibition of NADPH oxidase is effective in inhibiting suicidal NETosis in vitro, recent studies on experimental mouse models of SLE (54) and gout (55), both of which lack NADPH oxidase, show more severe diseases. In line with these studies, patients with chronic granulomatos disease, with defective NADPH oxidase, have a greater incidence of autoimmune diseases (63). Lowdensity granulocytes are the neutrophil subpopulation that release the most NETs both in AAV (28) and SLE (32). LDG NETs from SLE patients have been shown to be enriched for mtDNA and formation of these NETs can be inhibited in vitro with the mitochondrial ROS inhibitor MitoTEMPO (51). Interestingly, treatment of MLR/lpr mice with MitoTEMPO limits release of mtDNA NETs and reduces disease severity (51). Thus, targeting these NETs might also be an interesting approach in AAV. Regarding PAD4, which was shown to be a good target in various mouse models to limit NET-mediated inflammation, it was shown in the same study as for NADPH oxidase that PAD4 ${ }^{--}$mice developed worse disease than control mice (54). It appears that a certain amount of ROS signalling and NETosis is important to limit inflammation, while excessive NETs can instead cause disease. A recent example of compounds tested in vitro relevant to this aspect is the tetrahydroisoquinolines 
that can inhibit NETosis without interfering with ROS production (64). These were shown to inhibit both spontaneous and PMA-induced NETosis in neutrophils from SLE patients.

DNase I, which efficiently degrades DNA and thus degrades NETs, is already being used clinically and has proven to be safe. However, a phase 1 clinical study of DNase I in SLE patients did not show any effect on double-stranded DNA autoantibody production, inflammatory markers, or disease severity (65). When applied in a mouse model of antiGBM-induced glomerulonephritis, DNase I rescued mice from haematuria, but not proteinuria (50). It appears that components of NETs such as histones and neutrophil elastase can still harm the vasculature after DNase I treatment (66). It is worth noting that the complement fragment C5a can also induce NETosis (11), and a recent randomised controlled trial in AAV patients showed that the C5a receptor inhibitor avacopan had positive effects on disease activity (67).

\section{Conclusion}

Several studies have taken different approaches to studying the role of NETs in vasculitis, including in vitro functional studies, drug-induced autoimmunity, and animal models, and these have proposed NETs to constitute a source of autoantibodies and to promote vascular damage. These events can be avoided or reversed by inhibiting NETosis, blocking the proteins that are present in NETs, or by clearing NETs that have already formed. It would, however, be valuable for our understanding of NETs in vasculitis to learn more about why some animal models of autoimmune disease with impaired NETosis show aggravation of disease. 
- There are signs of increased NETosis in tissue and blood samples from vasculitis patients.

285

- Neutrophils from vasculitis patients are more prone to undergo NETosis in vitro, and serum

286

287

288

289

290

291

292

293

294

295

296

297

298

299 Conflicts of interest

$300 \quad$ None

\section{Acknowledgements}

None and autoantibodies from these patients induce NETosis in neutrophils from healthy controls.

- NETosis can induce endothelial damage both directly and indirectly, but NETosis may also be protective under some circumstances.

- There are several pharmacological approaches to alter NETosis, but such treatments, like the NETs themselves, might prove to be double-edged swords.

\section{Financial support and sponsorship}

This review was funded by the Ingrid Asps Foundation, the Swedish Society of Nephrology, and the Swedish Renal Foundation. 


\section{References}

303 1. Brinkmann V, Reichard U, Goosmann C, Fauler B, Uhlemann Y, Weiss DS, et al. 304 Neutrophil extracellular traps kill bacteria. Science. 2004;303:1532-5.

305 2. Jorch SK, Kubes P. An emerging role for neutrophil extracellular traps in 306 noninfectious disease. Nat Med. 2017;23:279-87.

307 3. Kessenbrock K, Krumbholz M, Schonermarck U, Back W, Gross WL, Werb Z, et al. 308 Netting neutrophils in autoimmune small-vessel vasculitis. Nat Med. 2009;15:623-5.

309 4. Fuchs TA, Abed U, Goosmann C, Hurwitz R, Schulze I, Wahn V, et al. Novel cell 310 death program leads to neutrophil extracellular traps. J Cell Biol. 2007;176:231-41.

311 5. Papayannopoulos V, Metzler KD, Hakkim A, Zychlinsky A. Neutrophil elastase and 312 myeloperoxidase regulate the formation of neutrophil extracellular traps. J Cell Biol. 2010;191:677-91.

314 6. Wang Y, Li M, Stadler S, Correll S, Li P, Wang D, et al. Histone hypercitrullination 315 mediates chromatin decondensation and neutrophil extracellular trap formation. J Cell Biol. $316 \quad 2009 ; 184: 205-13$.

317 7. Urban CF, Ermert D, Schmid M, Abu-Abed U, Goosmann C, Nacken W, et al. 318 Neutrophil extracellular traps contain calprotectin, a cytosolic protein complex involved in 319 host defense against Candida albicans. PLoS pathogens. 2009;5:e1000639.

320 8. Mohanty T, Sjogren J, Kahn F, Abu-Humaidan AH, Fisker N, Assing K, et al. A 321 novel mechanism for NETosis provides antimicrobial defense at the oral mucosa. Blood. $322 \quad 2015 ; 126: 2128-37$.

323 9. Goldmann O, Medina E. The expanding world of extracellular traps: not only neutrophils but much more. Frontiers in immunology. 2012;3:420. 
10. Morshed M, Hlushchuk R, Simon D, Walls AF, Obata-Ninomiya K, Karasuyama H, et al. NADPH oxidase-independent formation of extracellular DNA traps by basophils. J Immunol. 2014;192:5314-23.

11. Yousefi S, Mihalache C, Kozlowski E, Schmid I, Simon HU. Viable neutrophils release mitochondrial DNA to form neutrophil extracellular traps. Cell Death Differ. 2009;16:1438-44.

12. Pilsczek FH, Salina D, Poon KK, Fahey C, Yipp BG, Sibley CD, et al. A novel mechanism of rapid nuclear neutrophil extracellular trap formation in response to Staphylococcus aureus. J Immunol. 2010;185:7413-25.

13. Rochael NC, Guimaraes-Costa AB, Nascimento MT, DeSouza-Vieira TS, Oliveira MP, Garcia e Souza LF, et al. Classical ROS-dependent and early/rapid ROS-independent release of Neutrophil Extracellular Traps triggered by Leishmania parasites. Scientific reports. 2015;5:18302.

14. Jennette JC, Falk RJ, Bacon PA, Basu N, Cid MC, Ferrario F, et al. 2012 revised International Chapel Hill Consensus Conference Nomenclature of Vasculitides. Arthritis Rheum. 2013;65:1-11.

15. Gross WL, Trabandt A, Reinhold-Keller E. Diagnosis and evaluation of vasculitis. Rheumatology (Oxford). 2000;39:245-52.

16. Soderberg D, Segelmark M. Neutrophil Extracellular Traps in ANCA-Associated 344 Vasculitis. Frontiers in immunology. 2016;7:256.

345 17. Yoshida M, Sasaki M, Sugisaki K, Yamaguchi Y, Yamada M. Neutrophil extracellular trap components in fibrinoid necrosis of the kidney with myeloperoxidaseANCA-associated vasculitis. Clinical kidney journal. 2013;6:308-12. 

trap formation is associated with autophagy-related signalling in ANCA-associated vasculitis. Clin Exp Immunol. 2015;180:408-18.

19. O'Sullivan KM, Lo CY, Summers SA, Elgass KD, McMillan PJ, Longano A, et al. Renal participation of myeloperoxidase in antineutrophil cytoplasmic antibody (ANCA)associated glomerulonephritis. Kidney Int. 2015;88:1030-46.

20. Nakazawa D, Tomaru U, Yamamoto C, Jodo S, Ishizu A. Abundant neutrophil extracellular traps in thrombus of patient with microscopic polyangiitis. Frontiers in immunology. 2012;3:333.

21. Abreu-Velez AM, Smith JG, Jr., Howard MS. Presence of neutrophil extracellular traps and antineutrophil cytoplasmic antibodies associated with vasculitides. North American journal of medical sciences. 2009;1:309-13.

22. Sangaletti S, Tripodo C, Chiodoni C, Guarnotta C, Cappetti B, Casalini P, et al.

Neutrophil extracellular traps mediate transfer of cytoplasmic neutrophil antigens to myeloid dendritic cells toward ANCA induction and associated autoimmunity. Blood. 2012;120:300718.

** 23. Carmona-Rivera C, Purmalek MM, Moore E, Waldman M, Walter PJ, Garraffo HM,

et al. A role for muscarinic receptors in neutrophil extracellular trap formation and levamisole-induced autoimmunity. JCI insight. 2017;2:e89780.

This article shows that levamisole induces NETosis via muscarinic receptors. This could thus constitute a novel therapeutic approach. This article also supports the notion that the presence of autoantibodies in drug-induced autoimmunity depends on NET formation. linkage between microscopic polyangiitis and thrombosis via neutrophil extracellular traps. 
* 25. Takeuchi H, Kawasaki T, Shigematsu K, Kawamura K, Oka N. Neutrophil extracellular traps in neuropathy with anti-neutrophil cytoplasmic autoantibody-associated microscopic polyangiitis. Clin Rheumatol. 2017;36:913-7.

This is the first study to report the presence of NETs in nerve biopsies from vasculitis patients.

26. Soderberg D, Kurz T, Motamedi A, Hellmark T, Eriksson P, Segelmark M. Increased levels of neutrophil extracellular trap remnants in the circulation of patients with small vessel vasculitis, but an inverse correlation to anti-neutrophil cytoplasmic antibodies during remission. Rheumatology (Oxford). 2015;54:2085-94.

* 27. Surmiak M, Hubalewska-Mazgaj M, Wawrzycka-Adamczyk K, Szczeklik W, Musial J, Brzozowski T, et al. Neutrophil-related and serum biomarkers in granulomatosis with polyangiitis support extracellular traps mechanism of the disease. Clin Exp Rheumatol. 2016;34:S98-104.

This study presents several molecules, including NETs, that are elevated in vascultis patients during active disease.

28. Grayson PC, Carmona-Rivera C, Xu L, Lim N, Gao Z, Asare AL, et al. NeutrophilRelated Gene Expression and Low-Density Granulocytes Associated With Disease Activity and Response to Treatment in Antineutrophil Cytoplasmic Antibody-Associated Vasculitis. Arthritis Rheumatol. 2015;67:1922-32.

29. Ohlsson SM, Ohlsson S, Soderberg D, Gunnarsson L, Pettersson A, Segelmark M, et al. Neutrophils from vasculitis patients exhibit an increased propensity for activation by antineutrophil cytoplasmic antibodies. Clin Exp Immunol. 2014;176:363-72.

30. Sur Chowdhury C, Giaglis S, Walker UA, Buser A, Hahn S, Hasler P. Enhanced neutrophil extracellular trap generation in rheumatoid arthritis: analysis of underlying signal 
transduction pathways and potential diagnostic utility. Arthritis research \& therapy. 2014;16:R122.

31. Khandpur R, Carmona-Rivera C, Vivekanandan-Giri A, Gizinski A, Yalavarthi S, Knight JS, et al. NETs are a source of citrullinated autoantigens and stimulate inflammatory responses in rheumatoid arthritis. Science translational medicine. 2013;5:178ra40.

32. Villanueva E, Yalavarthi S, Berthier CC, Hodgin JB, Khandpur R, Lin AM, et al. Netting neutrophils induce endothelial damage, infiltrate tissues, and expose immunostimulatory molecules in systemic lupus erythematosus. J Immunol. 2011;187:53852.

* 33. Nishide M, Nojima S, Ito D, Takamatsu H, Koyama S, Kang S, et al. Semaphorin 4D inhibits neutrophil activation and is involved in the pathogenesis of neutrophil-mediated autoimmune vasculitis. Ann Rheum Dis. 2017.

This study suggests a mechanism that could be important for the increased rate of NETosis in vasculitis patients with respect to neutrophil and endothelial cell interactions.

34. Nakazawa D, Shida H, Tomaru U, Yoshida M, Nishio S, Atsumi T, et al. Enhanced formation and disordered regulation of NETs in myeloperoxidase-ANCA-associated microscopic polyangiitis. J Am Soc Nephrol. 2014;25:990-7.

* 35. Ma YH, Ma TT, Wang C, Wang H, Chang DY, Chen M, et al. High-mobility group box 1 potentiates antineutrophil cytoplasmic antibody-inducing neutrophil extracellular traps formation. Arthritis research \& therapy. 2016;18:2.

This article shows that ANCAs induce NETosis after priming of neutrophils with HMGB1, which is interesting because the levels of HMGB1 correlate with disease activity in other studies. 
* 36. Sha LL, Wang H, Wang C, Peng HY, Chen M, Zhao MH. Autophagy is induced by anti-neutrophil cytoplasmic Abs and promotes neutrophil extracellular traps formation. Innate immunity. 2016;22:658-65.

This study provides information on the signalling pathway involved during ANCA-induced NETosis.

* 37. Shida H, Nakazawa D, Tateyama Y, Miyoshi A, Kusunoki Y, Hattanda F, et al. The Presence of Anti-Lactoferrin Antibodies in a Subgroup of Eosinophilic Granulomatosis with Polyangiitis Patients and Their Possible Contribution to Enhancement of Neutrophil Extracellular Trap Formation. Frontiers in immunology. 2016;7:636.

In additon to the already established knowledge that PR3-ANCA and MPO-ANCA can induce NETosis, this article shows that lactoferrin-ANCA can also induce NETosis.

* 38. Kraaij T KS, Bakker J, Brunini F, Pusey C, Scherer HU, Toes REM, Rabelink T, van, Kooten C TO. NET-Inducing Capacity Is a Biomarker in ANCA-Associated Vasculitis Independent of ANCA Antibodies [abstract]. Arthritis Rheumatol. 2016;68 (suppl 10).

This study shows that components in serum other than ANCA are capable of inducing NETosis because they observed no differences between whole serum and IgG-depleted serum in this regard.

* 39. Natorska J, Zabczyk M, Siudut J, Krawiec P, Mastalerz L, Undas A. Neutrophil extracellular traps formation in patients with eosinophilic granulomatosis with polyangiitis: association with eosinophilic inflammation. Clin Exp Rheumatol. 2017;35 Suppl 103:27-32.

Serum from EGPA patients induces more NETosis than serum from healthy controls. There were no differences between ANCA-positive and ANCA-negative serum, suggesting other components in serum induce NETosis

* 40. Yoshida M, Yamada M, Sudo Y, Kojima T, Tomiyasu T, Yoshikawa N, et al. Myeloperoxidase anti-neutrophil cytoplasmic antibody affinity is associated with the 
445 formation of neutrophil extracellular traps in the kidney and vasculitis activity in 446 myeloperoxidase anti-neutrophil cytoplasmic antibody-associated microscopic polyangiitis. 447 Nephrology (Carlton). 2016;21:624-9.

448 This article strengthens the connection between ANCA affinity and NET-inducing capacity 449 because there is a correlation between the presence of NETs in kidney biopsies and ANCA 450 affinity.

451 41. Aleman OR, Mora N, Cortes-Vieyra R, Uribe-Querol E, Rosales C. Differential Use 452 of Human Neutrophil Fcgamma Receptors for Inducing Neutrophil Extracellular Trap 453 Formation. Journal of immunology research. 2016;2016:2908034. 
* 42. Kraaij T, Tengstrom FC, Kamerling SW, Pusey CD, Scherer HU, Toes RE, et al. A novel method for high-throughput detection and quantification of neutrophil extracellular traps reveals ROS-independent NET release with immune complexes. Autoimmunity reviews. 2016;15:577-84.

This study presents a novel sensitive approach to quantifiy NETs. This is a good approach because no NETs are being washed away before quantification.

* 43. Aleyd E, Al M, Tuk CW, van der Laken CJ, van Egmond M. IgA Complexes in Plasma and Synovial Fluid of Patients with Rheumatoid Arthritis Induce Neutrophil Extracellular Traps via FcalphaRI. J Immunol. 2016;197:4552-9.

This article contributes to the pathophysiological aspects of IgA because it shows that IgA immune complexes can induce NETosis.

44. Sandin C, Eriksson P, Segelmark M, Skogh T, Kastbom A. IgA- and SIgA anti-PR3 antibodies in serum versus organ involvement and disease activity in PR3-ANCA-associated vasculitis. Clin Exp Immunol. 2016;184:208-15.

45. Korkmaz B, Horwitz MS, Jenne DE, Gauthier F. Neutrophil elastase, proteinase 3, and cathepsin G as therapeutic targets in human diseases. Pharmacol Rev. 2010;62:726-59.

46. Kumar SV, Kulkarni OP, Mulay SR, Darisipudi MN, Romoli S, Thomasova D, et al. Neutrophil Extracellular Trap-Related Extracellular Histones Cause Vascular Necrosis in Severe GN. J Am Soc Nephrol. 2015;26:2399-413.

* 47. Pieterse E, Rother N, Garsen M, Hofstra JM, Satchell SC, Hoffmann M, et al. Neutrophil Extracellular Traps Drive Endothelial-to-Mesenchymal Transition. Arterioscler Thromb Vasc Biol. 2017;37:1371-9.

Endothelial cells are capable of phagocytising NETs. However, excessive amounts of NETs disrupt endothelial integrity and drive EndMT. 
48. Lin F, Wang N, Zhang TC. The role of endothelial-mesenchymal transition in development and pathological process. IUBMB life. 2012;64:717-23.

49. Wang $\mathrm{H}$, Wang $\mathrm{C}$, Zhao $\mathrm{MH}$, Chen M. Neutrophil extracellular traps can activate alternative complement pathways. Clin Exp Immunol. 2015;181:518-27.

* 50. Westhorpe CL, Bayard JE, O'Sullivan KM, Hall P, Cheng Q, Kitching AR, et al. In Vivo Imaging of Inflamed Glomeruli Reveals Dynamics of Neutrophil Extracellular Trap Formation in Glomerular Capillaries. Am J Pathol. 2017;187:318-31.

This article shows that shear stress plays an important role in the extent to which NETs will remain in the vessel or will be washed away and thus influences the damage that the NETs can cause.

** 51. Lood C, Blanco LP, Purmalek MM, Carmona-Rivera C, De Ravin SS, Smith CK, et al. Neutrophil extracellular traps enriched in oxidized mitochondrial DNA are interferogenic and contribute to lupus-like disease. Nat Med. 2016;22:146-53.

This article describes proinflammatory mechanisms of mitochondrial-derived NETs from LDGs and shows that LDGs from patients with SLE have an increased propensity to release mtDNA NETs. mtDNA NETs are also shown to be important in the development of SLE-like disease in mice because release of mtDNA NETs was reduced and disease ameliorated by targeting mtROS.

** 52. Nakazawa D, Kumar SV, Marschner J, Desai J, Holderied A, Rath L, et al. Histones and Neutrophil Extracellular Traps Enhance Tubular Necrosis and Remote Organ Injury in Ischemic AKI. J Am Soc Nephrol. 2017;28:1753-68.

This study provides information on epithelial cell and neutrophil interactions with respect to necroinflammation, where histones from epithelial cells can induce NETs, which in turn cause epithelial cell death and the subsequent release of more histones. 
503

504

505

506

507

508

509

510

511

512

513

514

515

516

517

518

519

520

521

522

523

524

525

* 53. Jiang D, Muschhammer J, Qi Y, Kugler A, de Vries JC, Saffarzadeh M, et al. Suppression of Neutrophil-Mediated Tissue Damage-A Novel Skill of Mesenchymal Stem Cells. Stem Cells. 2016;34:2393-406.

Injection of AT-MSCs in a mouse model of immune complex vasculitis suppresses NETosis and vessel damage in the skin, suggesting this to be a potential therapeutic approach.

** 54. Kienhofer D, Hahn J, Stoof J, Csepregi JZ, Reinwald C, Urbonaviciute V, et al. Experimental lupus is aggravated in mouse strains with impaired induction of neutrophil extracellular traps. JCI insight. 2017;2.

This study shows that impaired NET formation leads to aggravation of disease in a mouse model of SLE. This suggests that NETs also contribute to limiting inflammatory processes, highlighting a complex role for NETs in inflammation.

55. Schauer C, Janko C, Munoz LE, Zhao Y, Kienhofer D, Frey B, et al. Aggregated neutrophil extracellular traps limit inflammation by degrading cytokines and chemokines. Nat Med. 2014;20:511-7.

* 56. Guimaraes-Costa AB, Rochael NC, Oliveira F, Echevarria-Lima J, Saraiva EM. Neutrophil Extracellular Traps Reprogram IL-4/GM-CSF-Induced Monocyte Differentiation to Anti-inflammatory Macrophages. Frontiers in immunology. 2017;8:523.

NETs can promote differentiation of macrophages into a phenotype of anti-inflammatory macrophages, which is important because such subpopulations generally are connected to the resolution of inflammation.

57. Mitroulis I, Kambas K, Chrysanthopoulou A, Skendros P, Apostolidou E, Kourtzelis I, et al. Neutrophil extracellular trap formation is associated with IL-1beta and autophagyrelated signaling in gout. PloS one. 2011;6:e29318. 

neutrophils are major inducers of type I IFN production in pediatric systemic lupus erythematosus. Science translational medicine. 2011;3:73ra20.

59. Balavoine AS, Glinoer D, Dubucquoi S, Wemeau JL. Antineutrophil Cytoplasmic Antibody-Positive Small-Vessel Vasculitis Associated with Antithyroid Drug Therapy: How Significant Is the Clinical Problem? Thyroid. 2015;25:1273-81.

60. Nakazawa D, Tomaru U, Ishizu A. Possible implication of disordered neutrophil extracellular traps in the pathogenesis of MPO-ANCA-associated vasculitis. Clinical and experimental nephrology. 2013;17:631-3.

* 61. Kusunoki Y, Nakazawa D, Shida H, Hattanda F, Miyoshi A, Masuda S, et al. Peptidylarginine Deiminase Inhibitor Suppresses Neutrophil Extracellular Trap Formation and MPO-ANCA Production. Frontiers in immunology. 2016;7:227.

This study provides evidence that NETs can bridge innate and adaptive immunity through the generation of ANCA, but also that ANCA is not sufficient to cause full-blown disease in this mouse model.

$541 * 62$. Lood C, Hughes GC. Neutrophil extracellular traps as a potential source of autoantigen in cocaine-associated autoimmunity. Rheumatology (Oxford). 2017;56:638-43. This article suggests that the development of autoantibodies in drug-induced autoimmunity relies on NET formation and that these autoantibodies in turn can induce NETosis. This could create a vicious circle.

546 63. Winkelstein JA, Marino MC, Johnston RB, Jr., Boyle J, Curnutte J, Gallin JI, et al. 547 Chronic granulomatous disease. Report on a national registry of 368 patients. Medicine 548 (Baltimore). 2000;79:155-69. 
* 64. Martinez NE, Zimmermann TJ, Goosmann C, Alexander T, Hedberg C, Ziegler S, et

551 Formation. Chembiochem : a European journal of chemical biology. 2017;18:888-93.

552 These compounds can inhibit NETosis without affecting ROS formation, which is interesting 553 from a therapeutic point of view.

554 65. Davis JC, Jr., Manzi S, Yarboro C, Rairie J, McInnes I, Averthelyi D, et al. 555 Recombinant human Dnase I (rhDNase) in patients with lupus nephritis. Lupus. 1999;8:68-76

556 66. Kolaczkowska E, Jenne CN, Surewaard BG, Thanabalasuriar A, Lee WY, Sanz MJ, et 557 al. Molecular mechanisms of NET formation and degradation revealed by intravital imaging 558 in the liver vasculature. Nature communications. 2015;6:6673.

559 67. Jayne DR, Bruchfeld AN, Harper L, Schaier M, Venning MC, Hamilton P, et al. 560 Randomized Trial of C5a Receptor Inhibitor Avacopan in ANCA-Associated Vasculitis. J 561 Am Soc Nephrol. 2017. 
Table 1. Neutrophil extracellular trap-inducing capacity of IgG and serum from vasculitis patients and HCs

\begin{tabular}{|c|c|c|c|c|c|c|}
\hline Ab/serum & Patients & Disease activity & $\begin{array}{l}\text { Primingl } \\
\text { activation }\end{array}$ & $\begin{array}{l}\text { Patients vs. } \\
\text { HCs }(+l-)\end{array}$ & Subgroup analyses & Ref \\
\hline $\lg G$ & AAV & Active and remission & TNF & + & NA & 3 \\
\hline $\lg G$ & MPA & Active & TNF & + & $\begin{array}{c}\text { NETosis correlated with } \\
\text { disease activity and antibody } \\
\text { affinity }\end{array}$ & 34 \\
\hline $\lg G$ & AAV & Active & HMGB1 & + & NA & 35 \\
\hline $\lg G$ & AAV & Active & TNF & + & NA & 36 \\
\hline Anti-Lactoferrin & EGPA & Active & PMA & + & $\begin{array}{l}\text { NETosis correlated with } \\
\text { disease activity }\end{array}$ & 37 \\
\hline Whole serum & AAV & Not available & No & + & $\begin{array}{l}\text { MPA serum induced more } \\
\text { NETosis than GPA serum }\end{array}$ & 38 \\
\hline Whole serum & EGPA & Remission & No & + & $\begin{array}{l}\text { MPO-ANCA+ serum induced } \\
\text { more NETosis than PR3- } \\
\text { ANCA }^{+} \text {and ANCA } \text { An }^{-} \text {serum }\end{array}$ & 39 \\
\hline $\begin{array}{l}\text { Serum from ANCA- } \\
\text { negative patients }\end{array}$ & EGPA & Remission & No & $\begin{array}{c}+ \\
\text { (vs. whole serum) }\end{array}$ & NA & 39 \\
\hline IgG-depleted serum & AAV & Not available & No & $\begin{array}{c}+ \\
\text { (vs. whole serum) }\end{array}$ & $\begin{array}{c}\text { No difference in NETosis } \\
\text { between IgG-depleted serum } \\
\text { and whole serum }\end{array}$ & 38 \\
\hline
\end{tabular}

+, increased NETosis; NA, not available; ANCA, anti-neutrophil cytoplasmic autoantibody; AAV, ANCA-associated vasculitis; MPA, myeloperoxidase; EGPA, eosinophilic granulomatosis with polyangiitis; HMGB1, high mobility group box 1; TNF, tumour necrosis factor; PMA, phorbol 12-myristate 12-acetate; HCs, healthy controls; GPA, granulomatosis with polyangiitis; PR3, proteinase 3 
Table 2. Therapeutic approaches that inhibit or reduce the effect of NETs on vascular damage

\begin{tabular}{|c|l|l|c|}
\hline Therapy & \multicolumn{1}{|c|}{ Agent/approach } & \multicolumn{1}{c|}{ Experimental setup } & Ref \\
\hline $\begin{array}{c}\text { Neutrophil } \\
\text { clearance }\end{array}$ & $\begin{array}{l}\text { AT-MSC injection, } \\
\text { clearing via phagocytosis }\end{array}$ & $\begin{array}{l}\text { Mouse model, BSA/anti-BSA } \\
\text { immune complex-mediated vasculitis }\end{array}$ & 53 \\
\hline C5aR inhibitor & Avacopan & $\begin{array}{l}\text { Randomised controlled trial in human } \\
\text { AAV, phase 2 }\end{array}$ & 67 \\
\hline $\begin{array}{c}\text { Histone } \\
\text { neutralisation }\end{array}$ & $\begin{array}{l}\text { Anti-histone antibodies or } \\
\text { Heparin or } \\
\text { Activated protein C }\end{array}$ & $\begin{array}{l}\text { Mouse model, sheep anti-rat GBM } \\
\text { serum-induced glomerular vasculitis }\end{array}$ & 46 \\
\hline PAD inhibitor & Cl-amidine & $\begin{array}{l}\text { Mouse model, sheep anti-rat GBM } \\
\text { serum-induced glomerular vasculitis }\end{array}$ & 46 \\
\hline PAD inhibitor & Cl-amidine & Mouse model, post-ischemic AKI & 52 \\
\hline DNA degradation & DNase I & $\begin{array}{l}\text { Mouse model, anti-GBM- } \\
\text { induced glomerular vasculitis }\end{array}$ & 50 \\
\hline ROS scavenging & $\begin{array}{l}\text { AT-MSC injection, ROS } \\
\text { scavenging via SOD3 }\end{array}$ & $\begin{array}{l}\text { Mouse model, BSA/anti-BSA } \\
\text { immune complex-mediated vasculitis }\end{array}$ & 53 \\
\hline mtROS inhibitor & MitoTEMPO & $\begin{array}{l}\text { Mouse model, spontaneous } \\
\text { development of SLE-like disease }\end{array}$ & 51 \\
\hline
\end{tabular}

mtROS, mitochondrial reactive oxygen species; PAD, peptidyl arginine deiminase;

AT-MSC, adipocyte tissue-derived mesenchymal stem cell; SOD3, superoxide dismutase 3;

SLE, systemic lupus erythematosus; GBM, glomerular basement membrane; AKI; acute kidney injury;

BSA, bovine serum albumin; AAV, ANCA-associated vasculitis 
